\title{
DIGITAL PUMPS IN SPEED-CONTROLLED SYSTEMS - AN ENERGY STUDY FOR A LOADER CRANE APPLICATION
}

\author{
Samuel Kärnell*, Amy Rankka, Alessandro Dell’Amico, Liselott Ericson \\ Division of Fluid and Mechatronic Systems, Linköping University, 58183 Linköping, Sweden \\ * Corresponding author: Tel.: +46 700 896196; E-mail address: samuel.karnell@liu.se
}

\begin{abstract}
Imagine a system with a pump driven by a speed-controlled electric motor. What and how much can be gained by using a pump with discretely variable displacement instead of a conventional fixed pump in such a system? This question is the focus in this paper, in which a simulation study based on a drive cycle for a loader crane is presented. The results indicate that the system efficiency from inverter input to pump output can increase by a few percentages. This might be considered small in relation to the increasing complexity that comes with discrete displacement. However, the results also show that a system with discrete displacement substantially reduces torque and cooling requirements on the electric motor. The required maximum torque can be reduced by 30 to $50 \%$ and the motor can generate up to $40 \%$ less heat since it can work in more efficient conditions. These potential benefits will be obtained with only a few discrete displacement settings available.
\end{abstract}

Keywords: Digital pumps, Loader crane, Speed-controlled system

\section{INTRODUCTION}

There is an increasing interest in implementing electric drives in mobile machines. A consequence is that speed-controlled systems are receiving more attention. Such systems have been used in industrial applications for some years, and several pump suppliers are offering solutions with fixed pumps and variable pumps as well as so-called dual-displacement pumps that are basically variable displacement pumps but with only two pre-set displacement settings [1][2][3]. Arguments for having adjustable displacement in speed-controlled systems are that the electric motor can be downsized and that the total efficiency can be increased.

When it comes to systems with discrete displacement pumps, several researchers have studied the performance of novel architectures, see e.g. [4][5][6]. However, these studies use fixed-speed prime movers and mainly focus on the system architecture, unlike the focus of this study.

\subsection{Contributions}

The idea behind this paper is to quantify the amount of energy that can be saved and how the requirements on the electric motor changes when discretely variable displacement is used instead of fixed displacement in a speed-controlled system. This study does not consider how the pump should be controlled in real-time applications and it does not take dynamic aspects of the system into account.

The system boundary is set from the input of the frequency converter to the outlet of the pump, as illustrated in Figure 1. The flow from the pump as well as the pressure increase over it is always considered to be positive. This means that regeneration possibilities are not considered.

\subsection{Digital Pumps}

The title of this paper includes the term "digital pump", but there is no widely accepted definition of what a digital pump is. Some might think of the Artemis Digital Displacement pump, but that is not necessarily the type of pump considered here. In this paper, a digital pump is defined as a pump with discretely variable displacement that is built up from several pump elements. Each pump element can be either active or not. The principle layout is illustrated in Figure 1. 


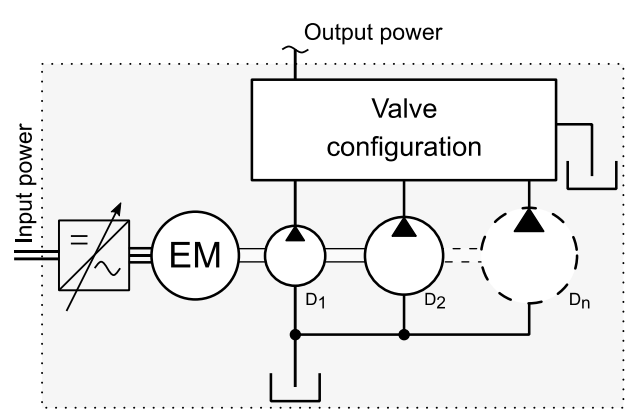

Figure 1: Principle layout of a digital pump with $n$ number of pump elements driven by one frequency controlled electric motor. The large box illustrates the system boundaries for this analysis.

Looking at Figure 1, it is reasonable to regard a digital pump as several stacked pumps, but there are other possible solutions. For example, some types of piston pumps can be turned into pumps with a discrete number of displacement settings by grouping the pistons in the pump [7]. When grouping pistons, digital pumps do not have to be much larger than fixed pumps and cost-wise it is only the additional valve configuration and its control, which is required to switch between displacement settings, that differs. Therefore, digital pumps can be an interesting alternative to fixed pumps.

\section{Valve configuration}

There are different ways of realising the valve configuration in a digital pump. For example, through use of a check valve in combination with a 2-way valve for each pump element, as shown in Figure 2. Characteristics of different valve combinations are discussed in [7]. However, how they differ is not important for the analysis presented in this paper.

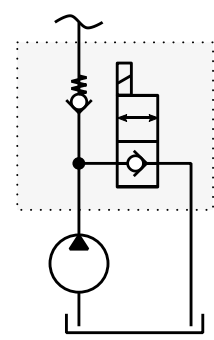

Figure 2: An example of a by-pass valve configuration.

\section{Scaling of pump elements}

The pump elements that the pump is built up from can be of arbitrary size and optimized for a specific case. However, another alternative is to use so-called binary scaling. That means that the second element is twice the size of the first, the third element is twice the size of the second and so on. Binary scaling results in an equally distributed displacement setting range. This is illustrated in Figure 3. All results presented in this paper are based on binary scaling.

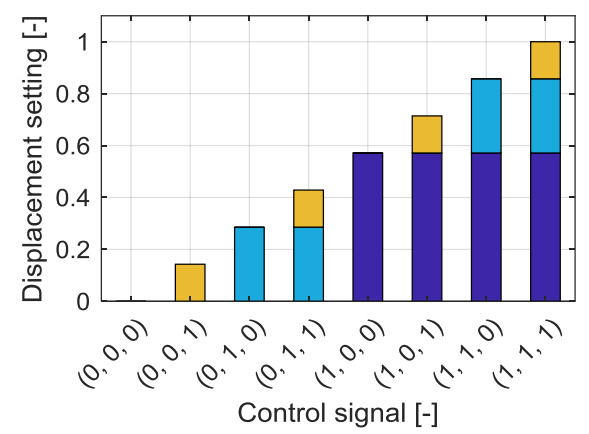

Figure 3: Control of a binary scaled digital pump with three pump elements.

\subsection{Synchronous machines}

There are many types of electric machines. However, permanent magnet synchronous machines (PMSM) are starting to get common in mobile systems due to their high efficiency and high power density. Therefore, this kind of electric machine has been considered in this analysis. The working range of a PMSM is usually divided into an intermittent region and a continuous region. An example of a typical working range is illustrated in Figure 4. As a rule of thumb, when dimensioning the motor for a drive cycle, the point where the root mean square of the torque meets the root mean square of the speed should be within the continuous region [8]. However, the size of the continuous region is highly dependent on the cooling capacity. Better cooling means higher continuous torque. The peak torque of the motor is principally defined by its physical size.

In addition to the intermittent and continuous region, a flux weakening region is also illustrated in Figure 4. Flux weakening must be achieved at speeds above the rated speed to reduce the back 
emf voltage, which increases with speed. Flux weakening costs energy, which means reduced efficiency. Nevertheless, in this study the rated speed is never exceeded.

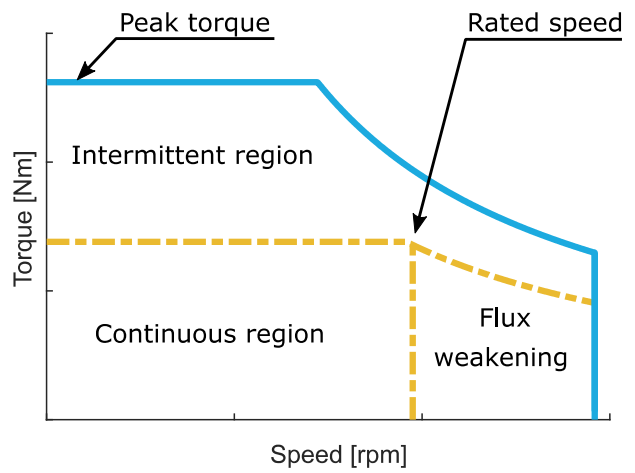

Figure 4: Example of working range for a liquid cooled synchronous motor.

\section{SIMULATION MODEL}

The simulation model that has been developed for this analysis is static. The input to the model is pressure and flow and from this, the required electric power is calculated. An overview of the model is illustrated in Figure 5.

\subsection{Model initialisation}

Before running the model, some parameters are initialised, primarily pump displacement, peak torque for the motor and inverter power rating. The parameters are based on the drive cycle and the rated motor speed. Throughout this analysis, the rated speed is set to $2400 \mathrm{rpm}$. The required pump displacement is calculated from the highest flow in the cycle and the rated speed. To compensate for the flow losses, the displacement is iteratively increased until it fulfils the required flow in the cycle. For the torque dimensioning, two different initialization methods have been used: one where the torque is calculated from the corner power and one where it is based on the maximum cycle power. The different power levels are shown later in Figure 10, where the drive cycle is presented.

\subsection{Control}

In this model, the most efficient displacement setting is used at all times. All possible displacement settings are evaluated for each point in the drive cycle and the one with the lowest losses is chosen. Restrictions are set to avoid speeds over the rated speed as well as torque over the peak torque. This control is inappropriate in a real system since it means a lot of switchings and requires much computational power. However, it gives the best possible energy efficiency, assuming switching losses due to compression and decompression of the oil are small.

\subsection{Pump model}

The pump model is based on look-up tables for the losses, which in turn are based on measurements provided by the manufacturer. The layout of the pump model is illustrated in the top block of Figure 5 and the efficiency map for the reference pump is shown in Figure 6.

The first steps in the pump model are to calculate the ideal speed and to determine the pressure for each pump element. This means that the inactive elements get the pressure that corresponds to their pressure drop over the bypass valve. For the active elements, the pressure drop is added up to the system pressure. The actual speed is then calculated by iterating the flow losses. When the actual speed is determined, the torque losses are calculated. Additionally, the actuation power required for the bypass valves is summed up to the power consumed by the system. The valves can be either normally pumping or normally bypassing. In these simulations normally pumping valves have been used, which means that no actuation power is required for an active pump element.

The losses from the look-up tables for the flow and torque losses are scaled with the displacement of the pump, which often is the case for analytical loss modelling in pumps [9].

\subsection{Electric motor model}

Like the pump model, the motor model is based on look-up tables, but with speed and relative torque as input variables and required input power as output. The model structure is shown in the middle block in Figure 5 and the efficiency of the reference motor is shown in Figure 7. 
When rescaling the motor parameters, the relative power losses are based on the relative torque. This assumption is based on efficiency maps in data sheets [8].

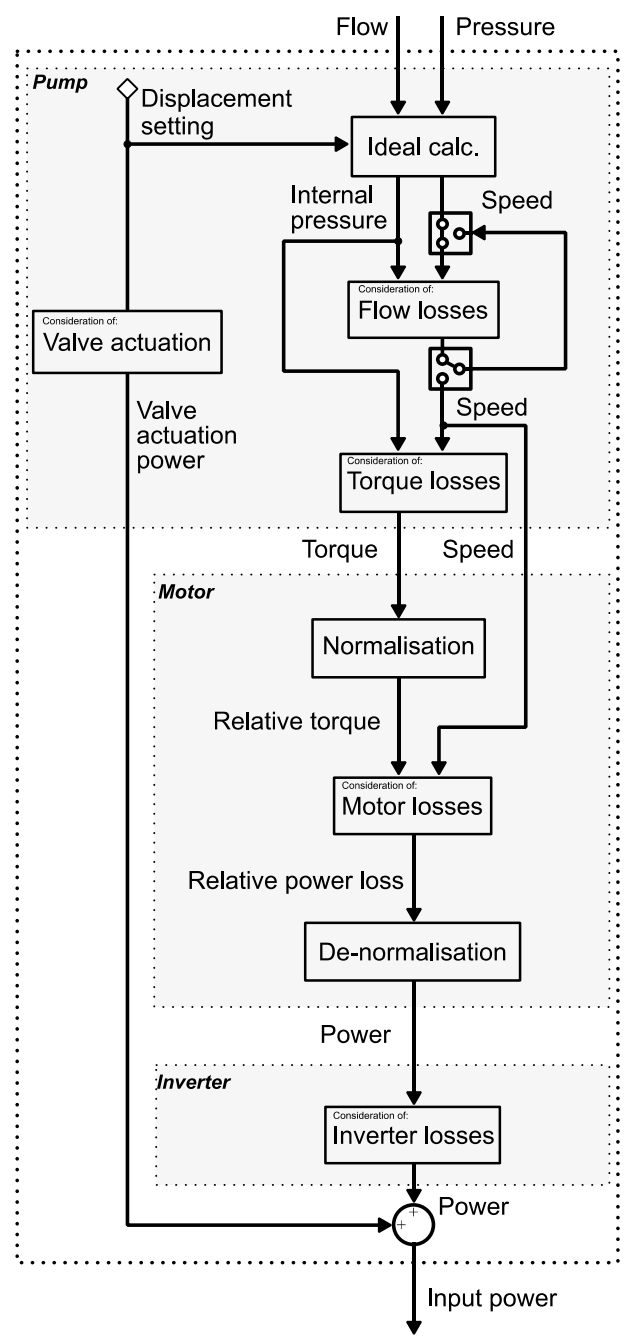

Figure 5: Layout of the simulation model. The arrows show the direction for the calculations, which is opposite to the energy flow. The model basically consists of three blocks; the pump, the motor and the inverter.

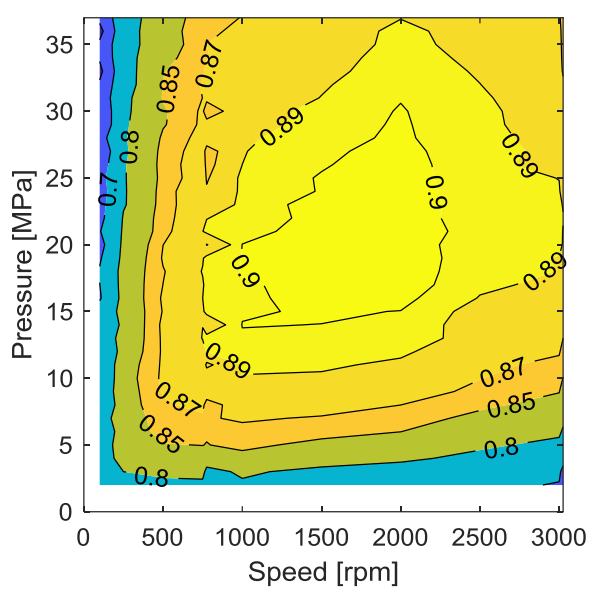

Figure 6: Measured total efficiency for the reference pump, which is a $34 \mathrm{cc}$ bent-axis pump.

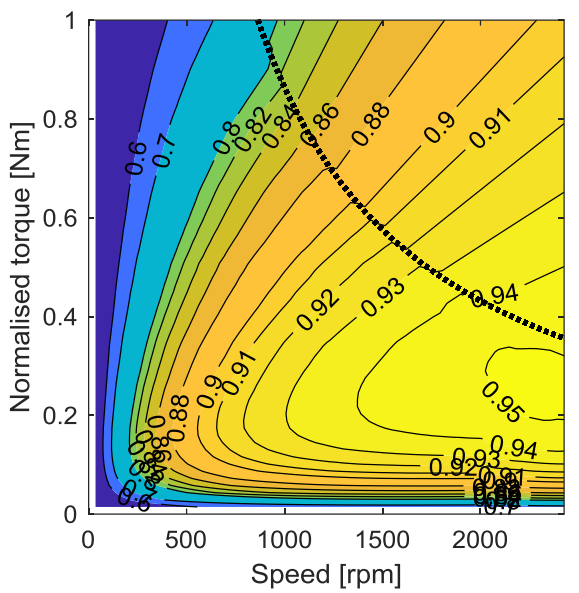

Figure 7: Provided synchronous motor efficiency with normalised torque. The dotted line represents the border between measurements and the described loss model.

In the motor data provided, measurements for the efficiency over a certain power were missing. To make the model more general, that region was filled with data from a simple loss model described in Equation 1:

$P_{\text {loss }}=T^{2} R+n^{\alpha} c$

$\mathrm{T}$ is the torque and $\mathrm{n}$ the speed. The other parameters are constants based on the provided measurements. The first term represents the copper losses and the second the core losses. The 
representation of the core losses is a result from lumping of parameters in the modified Steinmetz equation [10]. The efficiencies above the dotted line in Figure 7 are based on this loss model. However, note that these efficiencies imply a high cooling capacity and that they only will be reached in simulations where the motor is dimensioned for the cycle power.

\subsection{Inverter model}

The losses in the inverter are mainly conduction and switching losses. The simulations are based on data for a typical inverter [11], which is shown in Figure 8.

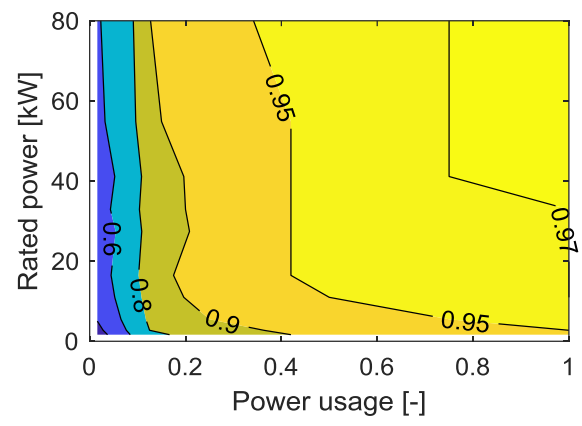

Figure 8: Inverter efficiency used in the model.

\section{DRIVE CYCLE}

As mentioned, the drive cycle corresponds to the input to the simulation model and is defined in terms of pump flow and pump pressure. It is based on long term field-measurements made on a crane. The pressure is measured, and the flow is derived from the cylinder positions. See [12] for more information about the drive cycle generation. Note that regeneration is not considered in this study since the required flow is defined as positive at all times.

Figure 9 shows histograms for the pressure, flow and power in the drive cycle. It can be seen that the crane is operating at very low power most of the time. The drive cycle is also represented in a pressure/flow-diagram in Figure 10.
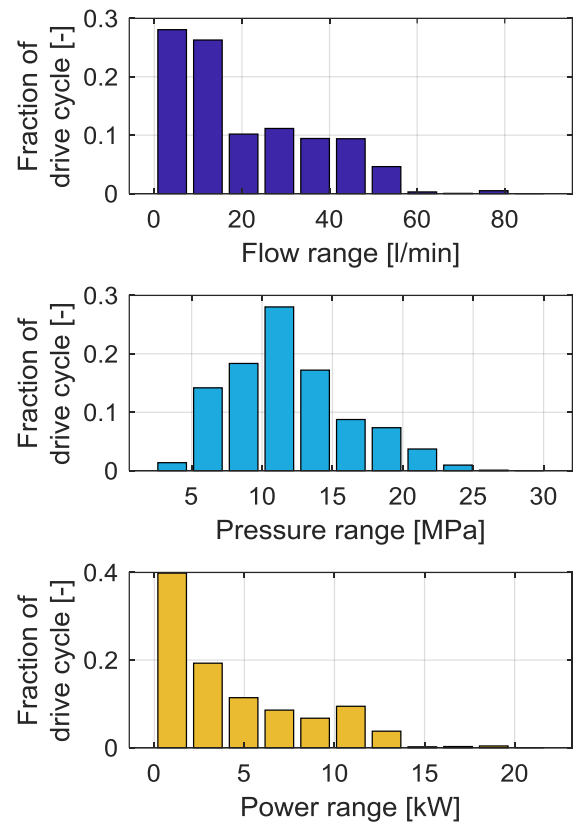

Figure 9: Flow, pressure and power distribution of the drive cycle.

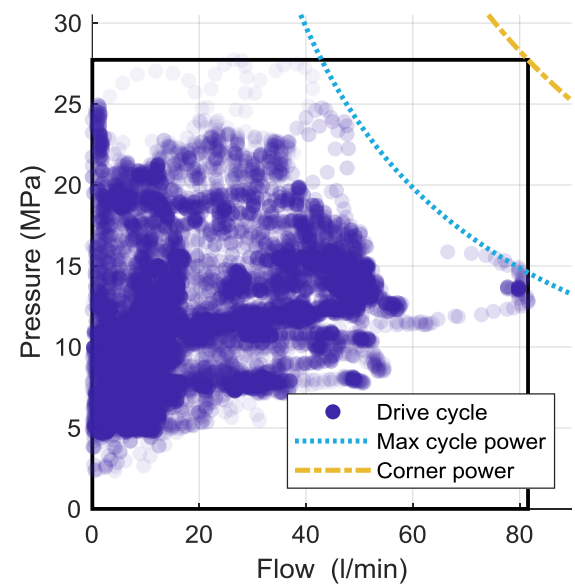

Figure 10: Pressure/flow-diagram of the drive cycle. The max cycle power-line shows the highest power used in the cycle and the corner power is the required power when using maximum flow at maximum pressure. 


\section{RESULTS}

As earlier stated, there are two main reasons to consider a digital pump; downsizing possibilities and energy savings. However, before going into the results, the general simulation setup is described.

\subsection{Simulation setup}

If nothing else is stated, the presented results are based on the following setup:

- Number of pump elements: 2.

- Rated motor speed: $2400 \mathrm{rpm}$.

- Valve size: $120 \mathrm{l} / \mathrm{min}$ at 10 bar pressure drop. The pressure drop is the same over the bypass line as for the line to the system for all pump elements.

- Valve configuration: normally pumping.

- Valve actuation power: $33 \mathrm{~W}$.

The efficiency of the system will also be affected by the size of the motor as well as the rated inverter power. As earlier mentioned, two different dimensioning methods are applied when dimensioning these components. One where the they are dimensioned strictly by the cycle. This method allows for downsizing of the electric motor and inverter. The other method is to dimension for the corner power. This means that the motor and the inverter must keep the same size for all simulated cases.

\subsection{Downsizing}

The downsizing possibilities for the analysed drive cycle, when only considering the peak torque, are illustrated in Figure 11. It shows that $67 \%$ of the reference peak torque is required to be able to run the whole cycle with 2 pump elements. With 3 pump elements it is instead 62 $\%$ and with 4 it is $56 \%$. This should be compared to a continuously variable pump where $53 \%$ of the torque is needed. Bear in mind that this relation is the same as the ratio between the maximum cycle power and the corner power illustrated in Figure 10.

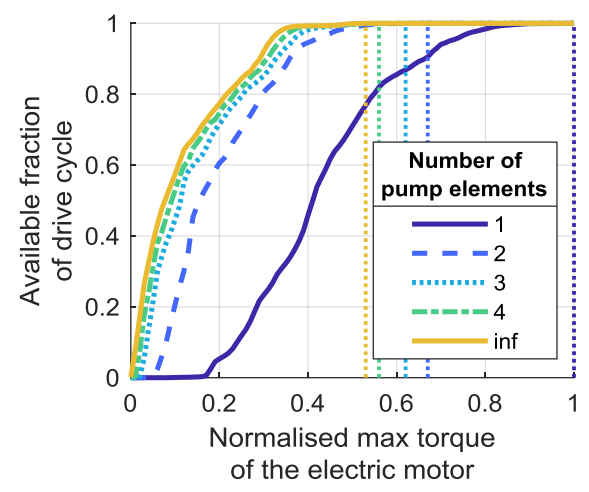

Figure 11: Drivable fraction of the drive cycle as a function of motor torque for different number of binary scaled pump elements. Inf corresponds to a continuously variable pump. The vertical lines are placed at the torque level where $100 \%$ of the cycle can be performed.

As mentioned in the introduction of the paper, it is not only the peak torque that is of interest when dimensioning the motor, but also the rms torque for the cycle, which can give an indication of the required continuous torque. The required peak torque and the rms torque for different numbers of pump elements are shown in Figure 12. The figure shows that the rms torque decreases slightly in relation to the peak torque when downsizing the motor. It also shows how the rms speed is increasing.

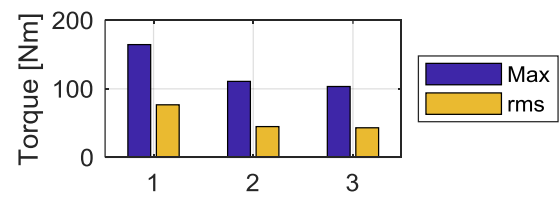

Number of pump elements [-]

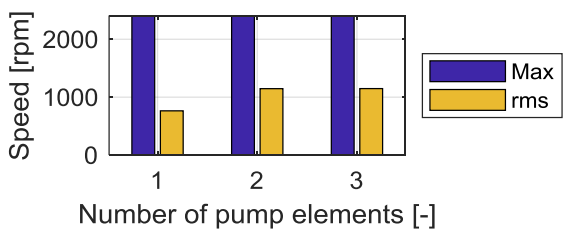

Figure 12: Relation between maximum values and root means square values torque and speed when using different numbers of pump elements. 


\subsection{Loss analysis}

The efficiency is defined as the output energy for the whole cycle divided by the input energy. Figure 13 shows how the efficiency varies with the valve size for different numbers of pump elements in both dimensioning cases. Note that the reference line is slightly higher than the curve for a digital pump with one pump element. The reason is the additional losses that comes with the bypass valve. When comparing the both dimensioning cases, one can first note that the efficiency is generally lower in the corner power case. This is mainly due to the fact that the inverter losses increase when the inverter is working at a lower relative power. Apart from that, the results are very similar. The total efficiency gains correspond to about two to three percentages for a $120 \mathrm{l} / \mathrm{min}$ valve.

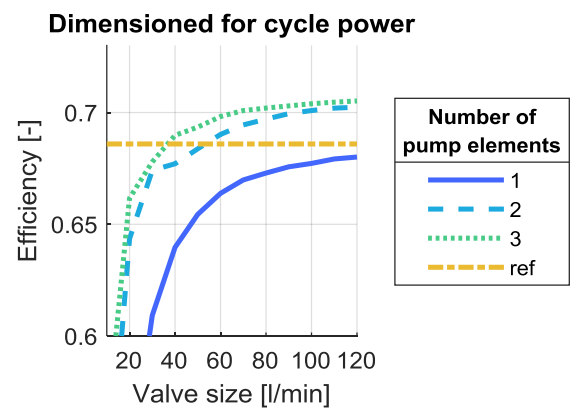

Dimensioned for corner power

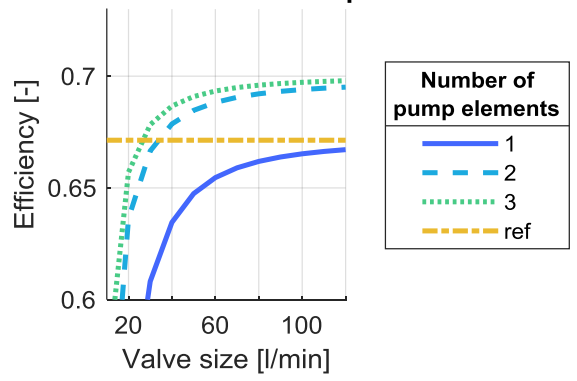

Figure 13: Efficiency for different valve sizes when varying the number of pump elements. The line labelled ref corresponds to a fixed pump without bypass functionality. In the upper figure components are dimensioned for the required cycle power whilst the lower shows components dimensioned for the corner power.
The loss distribution between the different components is illustrated in Figure 14. It shows that the introduction of more pump elements reduces the total losses by about $10 \%$. However, note that the motor losses can be reduced by up to $40 \%$.
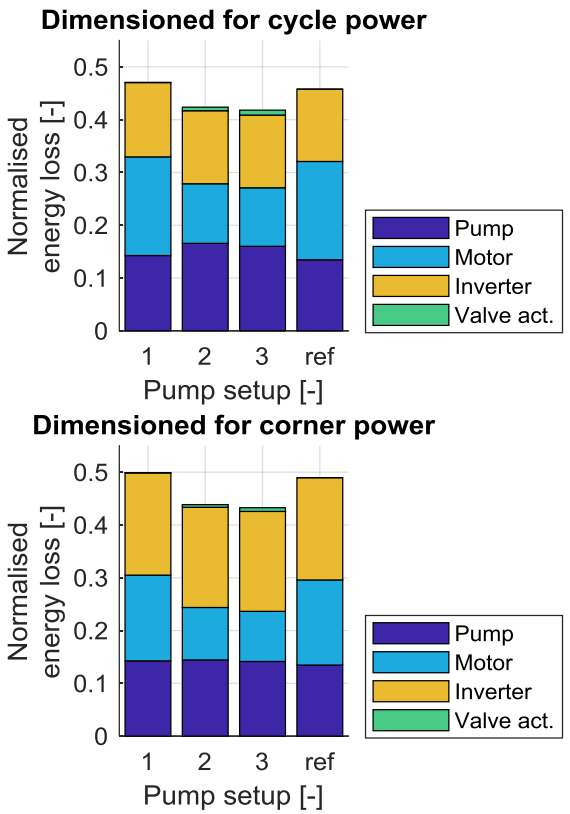

Figure 14: Energy losses in the different components over the full drive cycle. The losses are normalised with the output power, which is defined by the drive cycle and equal for all pump setups. The pressure drop over the valve configuration is included in the pump losses.

In Figure 15, it is illustrated how the working points for the motor changes when increasing the number of pump elements without downsizing the motor. It can be seen how the points are moving towards a more efficient region. The downsizing possibilities are also visible since the required torque is reduced when the number of pump elements is increased. If the motor is downsized, the results will be similar, but the efficiency maps will be rescaled and the motor will have to work in its high-power corner, where the losses are modelled according to Equation 1. 

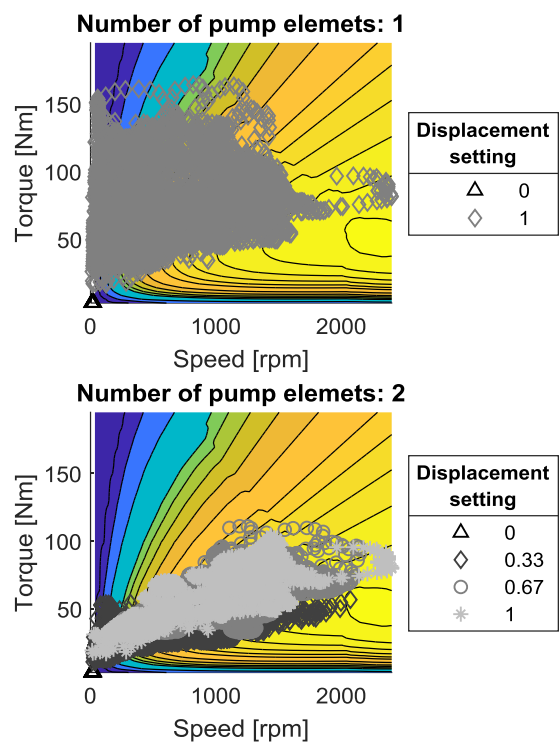

Number of pump elemets: 3

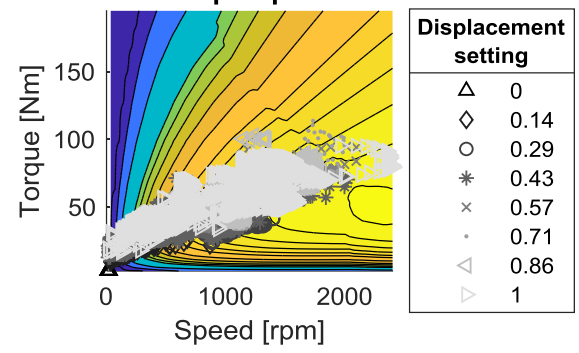

Figure 15: Drive cycle scattered over the motor efficiency map for different numbers of pump elements when dimensioning the components for the corner power.

\subsection{Drive cycle variations and generalisability}

The results presented so far are based on the specific drive cycle. However, it is of interest to see to what extent the results are applicable to other cases. To illustrate how sensitive the results are to variations in the drive cycle, random noise of different amplitudes was added to the cycle. Figure 16 shows how the efficiency varies with added noise. The implementation method of the noise signal is shown in Figure 17. The results indicate that the difference in efficiency between a digital and a fixed pump is almost independent of the noise. The inclination of the efficiency can be explained by that fact that the average power increases when adding noise this way since the output values are saturated between zero and the maximum cycle value.

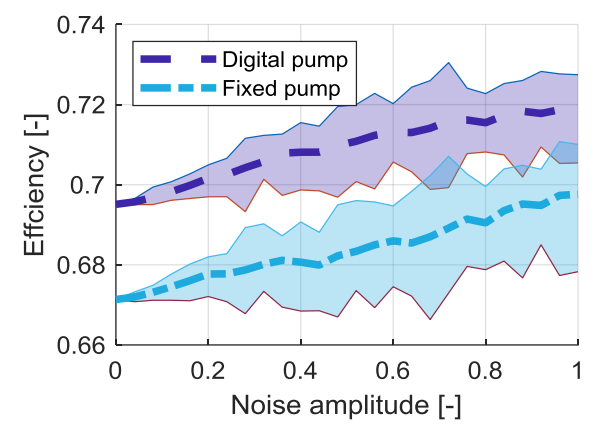

Figure 16: Sensitivity to changes in the drive cycle. 25 simulations were run for all analysed noise level amplitudes. The shaded areas corresponds to the efficiency variations and the lines shows the average efficiency. The implementation of the noise signal is shown in Figure 17.

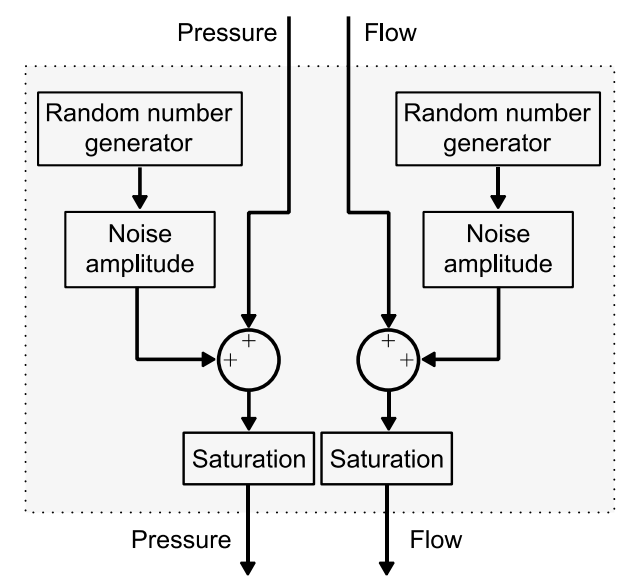

Figure 17: Implementation of the noise signal. The random number generator generates a number between the negative and positive maximum cycle value. This is multiplied by the noise amplitude factor and the product is added to the original signal. The saturation block limits the output to be between zero and the maximum cycle value. 
To determine if a drive cycle is applicable for a digital pump, the absolute power loss reductions for each work condition is of interest. Figure 18 shows the difference in power consumption between a digital pump with two pump elements dimensioned for the corner power and a fixed pump. It shows that the highest loss reduction possibilities are in the high-pressure/low-flow region, when the lowest displacement setting is in use. This means that drive cycles working much in that region will have high efficiency gain possibilities. This is because the motor does not have to work much in high-torque/low-speed conditions. However, when the highest displacement is in use, the losses will be slightly higher for a digital pump than a fixed because of the additional pressure drops over the additional valves.

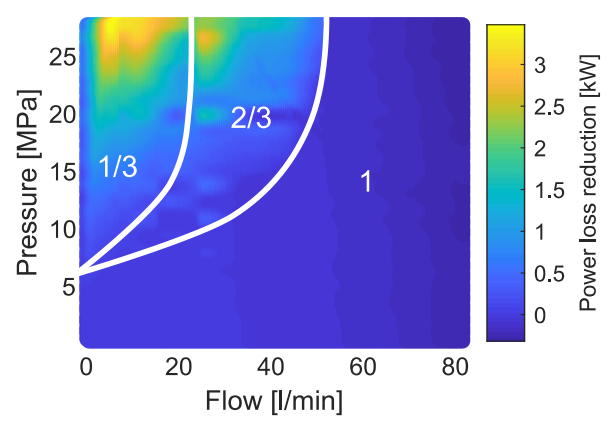

Figure 18: Difference in power consumption for a corner power dimensioned digital pump with two pump elements and a fixed pump. The figure also shows the most efficient displacement setting for each working condition.

\section{DISCUSSION}

The increase in efficiency for the analysed case is only a few percentages since the drive cycle mostly is in the moderate pressure region, where the difference in power losses are small, as illustrated in Figure 18. The impact will be greater for a drive cycle that rarely requires high flow but low flow at high pressure often. A related notice is that it, from an energy perspective, could be interesting to only have two available displacement settings since displacement settings in the higher region does not affect the efficiency much.
Nevertheless, this study shows that the total efficiency is not increased much, but when looking at energy losses, the numbers are larger, especially the energy losses in the motor, which can be reduced by about $30-40 \%$. This means that the lifetime of the motor can be increased, since it will be exposed to less heat. Alternatively, the cooling restrictions of the motor can be lowered. It has also been stated that the peak torque of the motor can be reduced if one allows dimensioning for maximum cycle power instead of corner power. However, note that these downsized motors must be able to run at high power in relation to their peak torque.

Anyway, smooth switching behavior is a criterion for digital pumps if they should be considered as relevant for loader cranes and similar applications. To achieve this, research is required. Furthermore, digital pumps must also be cheaper and/or have better efficiency than variable pumps since variable pumps allows for the same or even better performance than digital pumps can do.

\section{CONCLUSIONS}

It has been shown that the differences in potential loss reductions and downsizing possibilities are small between a digital pump with two and three pump elements. Regarding the total efficiency, it can be increased by a few percentages for the analysed drive cycle, but the potential benefits are higher for cycles that often requires low flow at high pressure. The results also show that even if the total efficiency is not increased much, the motor losses can be reduced substantially. The requirement of the peak torque can also be significantly reduced. This means that digital pumps mainly are relevant if downsizing of the electric motor is of interest or if the cooling requirements of it should be reduced, alternatively if the drive cycle often requires low flow at high pressure.

\section{ACKNOWLEDGEMENTS}

The authors would like to thank the Swedish Energy Agency, who are sponsoring the project STEALTH - Sustainable Electrified Load Handling, App. No 44427-1, of which this work is a part. 


\section{NOMENCLATURE}

$\begin{array}{ll}\mathrm{P}_{\text {loss }} & \text { Power loss }[\mathrm{W}] \\ \mathrm{T} & \text { Torque }[\mathrm{Nm}] \\ \mathrm{R} & \text { Lumped constant }[(\mathrm{sNm})-1] \\ \mathrm{n} & \text { Speed }[\mathrm{rad} / \mathrm{s}] \\ \alpha & \text { Constant }[-] \\ \mathrm{c} & \text { Lumped constant }[\mathrm{Nm}]\end{array}$

\section{REFERENCES}

[1] Moog Inc. (2017) Electrohydrostatic pump unit. CDL49052-en, Rev. D.

[2] Eaton (2015) Variable speed drive pump solution. E-PUIO-CC002-E.

[3] Parker Hannifin Corporation (2017) Drive Controlled Pump Effiziente hydraulische Antriebe mit System. HY11-3351/DE.

[4] Moorhead JR (1984) Saving energy with digital pump systems. Machine Design, 56(4): 40-44

[5] Heitzig S, Sgro S, Theissen H (2012) Energy efficiency of hydraulic systems with shared digital pumps. International journal of fluid power 13(3): 49-57

[6] Locateli C, Teixeira P, De Pieri E, Krus P, De Negri V (2014) Digital hydraulic system using pumps and on/off valves controlling the actuator. In 8th FPNI Ph. D symposium on fluid power, pages V001T01A009-V001T01A009. American Society of Mechanical Engineers.

[7] Kärnell S, Ericson L (2019) As Simple as Imaginable - an Analysis of Novel Digital Pump Concepts. 16th Scandinavian International Conference on Fluid Power

[8] Parker Hannifin Corporation (2018) Global Vehicle Motors GVM Series. PVD 3668 GB.

[9] Huhtala K, Vilenius M (1997) Comparison of steady state models of hydraulic pump. 5th Scandinavian International Conference on Fluid Power

[10] Reinert J, Brockmeyer A, De Doncker R. W (2001) Calculation of losses in ferro-and ferrimagnetic materials based on the modified Steinmetz equation. IEEE Transactions on Industry applications, 37(4), 1055-1061

[11] U.S. Department of Energy (2012) Adjustable Speed Drive Part-Load Efficiency. DOE/GO102012-3730

[12] Rankka A, Dell'Amico A, Krus P (2019) Drive Cycle Generation for a Hydraulic Loader Crane. The 16th Scandinavian International Conference on Fluid Power 